\title{
Design Principles for Engineering of Tissues from Human Pluripotent Stem Cells
}

\author{
Oriane B. Matthys ${ }^{1,2} \cdot$ Tracy A. Hookway $^{1} \cdot$ Todd C. McDevitt $^{1,3}$
}

Published online: 27 January 2016

(C) Springer International Publishing AG 2016

\begin{abstract}
Recent advances in human pluripotent stem cell (hPSC) technologies have enabled the engineering of human tissue constructs for developmental studies, disease modeling, and drug screening platforms. In vitro tissue formation can be generally described at three levels of cellular organization. Multicellular hPSC constructs are initially formed either with polymeric scaffold materials or simply via self-assembly and adhesive mechanisms. Heterotypic interactions within hPSC tissue constructs can be achieved by physically mixing independently differentiated cell populations or coaxed to simultaneously co-emerge from a common population of undifferentiated cells. Higher-order tissue architecture can be engineered by imposing external spatial constraints, such as molds and scaffolds, or depend upon cell-driven organization that exploits endogenous innate developmental mechanisms. The multicellular, heterogeneous, and highly organized structure of hPSC constructs ultimately dictates the resulting form and function of in vitro engineered human tissue models.
\end{abstract}

This article is part of the Topical Collection on Stem Cells and Nanotechnologies

Todd C. McDevitt

todd.mcdevitt@gladstone.ucsf.edu

1 The Gladstone Institute of Cardiovascular Disease, 1650 Owens St, San Francisco, CA 94158, USA

2 Graduate Program in Bioengineering, University of California Berkeley and University of California San Francisco, Berkeley/San Francisco, CA, USA

3 Department of Bioengineering and Therapeutic Sciences, University of California San Francisco, San Francisco, CA, USA
Keywords Human pluripotent stem cells - Tissue engineering · Organoids · Multicellular assembly · Heterogeneous cell populations · Tissue architecture

\section{Introduction}

Significant advances in pluripotent stem cell (PSC) biology over the past two decades afford many new and exciting opportunities for the engineering of human tissues from PSCs. The unique ability of PSCs to self-renew indefinitely in vitro and differentiate into cell types from all three germ layers makes them an attractive cell source for engineering a wide variety of tissues. Human PSCs were originally derived from the inner cell mass of in vitro fertilized blastocysts, hence referred to as "embryonic" stem cells (ESCs) [1]. Later studies found that reprogramming human somatic cells with transient expression of four exogenous transcription factors was sufficient to create "induced" pluripotent stem cells (iPSCs) [2,3]. The discovery of induced reprogramming and subsequent reproducibility by many other investigators in short order led to the widespread use and availability of diverse human PSC lines for research. Consequently, better-defined methods for reprogramming as well as an improved mechanistic understanding of pluripotency and differentiation have contributed to robust reagents and protocols and a greater consensus on differentiation and reprogramming standards.

Despite the successes in deriving PSCs in different manners, directed differentiation of human pluripotent stem cells (hPSCs) into specified cell types in an efficient and reproducible manner still remains a frequent challenge. Current differentiation protocols that largely mimic known developmental signaling molecules and pathways generally involve a series of steps to first direct differentiation toward a single germ layer and then yield a specific phenotype shortly thereafter. 
The continued development of protocols has made it possible to obtain fairly enriched or highly pure populations of hPSC differentiated cell types for tissue engineering constructs, including cardiomyocytes [4-6], neurons [7, 8], retinal pigment epithelium cells [9], liver hepatocytes [10, 11], and pancreatic endocrine cells $[12,13]$. In addition, several striking examples of various organoids derived from hPSCs in recent years provide an alternative strategy for direct engineering of tissues from human PSC sources [14-16].

Some of the primary defining characteristics of a tissue include the physical assembly of multiple cells, heterogeneity of cell phenotypes, and higher-order organization of tissue architecture. Functional tissues are inherently comprised of multiple cell populations that interact within and between populations as well as with their extracellular environment to dictate tissue form and function. These multicellular and heterogeneous cell mixtures are organized in threedimensional arrangements that span multiple scales and ultimately work cooperatively together to perform particular functions.

This review aims to define the key components and levels of organization of tissue formation for human PSCs and address the current limitations the field is facing with regards to the engineering of tissue constructs.

\section{Assembling hPSCs into Multicellular Constructs}

The first step in engineering any hPSC-derived tissue is to assemble the cells at a sufficiently high density in $3 \mathrm{D}$. Generally speaking, 3D hPSC multicellular constructs can be created either by seeding on or within a scaffold material or assembling the cells in a "scaffold-free" manner by relying on intrinsic intercellular adhesion mechanisms (Fig. 1a).

\section{Scaffold-Based hPSC Assembly}

Scaffolds for hPSC assembly are made from synthetic or naturally derived polymeric materials in the form of either highly porous constructs or encapsulating hydrogels. The primary intent of polymeric scaffolds is to provide physical support for cell survival and growth. Cell assembly onto scaffold materials is primarily mediated by cell-extracellular matrix (ECM) adhesions such as integrins, which are transmembrane proteins on the cell surface that recognize peptide sequences found within many ECM molecules, thereby allowing cells to anchor to the surrounding scaffold $[17,18]$.

Scaffold-based assembly of tissues enables precise control of many aspects of the microenvironment. Some scaffold fabrication parameters to be considered when designing synthetic materials are material choice, pore size, porosity, fiber diameter, fiber alignment, stiffness, etc., which may directly impact the ability of hPSCs to attach, grow, and differentiate into a desired tissue type [19, 20]. For example, poly-D-lysine-based scaffolds support the attachment and growth of hPSCs only when electrospun into microfibrous geometries. However, macroporous sponges of the same material fail to promote attachment and growth of hPSCs, indicating that physical parameters can be critical to achieving hPSC assembly and tissue formation [21]. To date, a variety of scaffold materials capable of supporting hPSC attachment and growth have been identified [20]; however, additional culture factors are

\section{A}
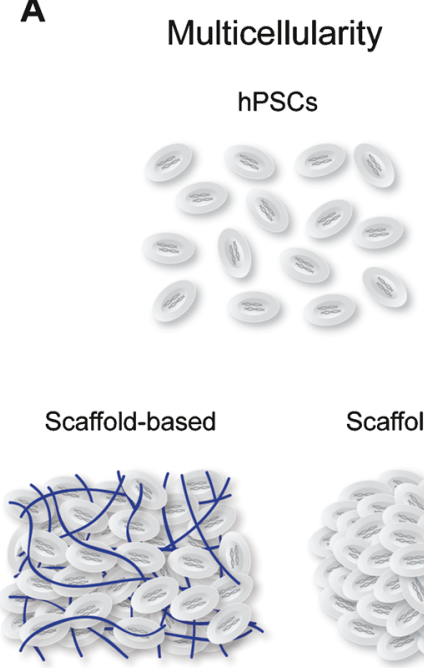

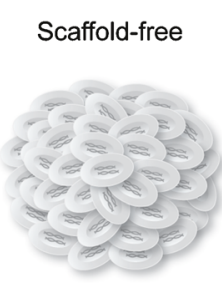

B

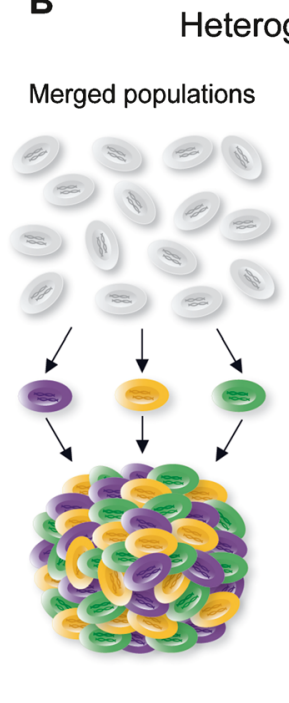

Fig. 1 Defining characteristics of tissue formation from human pluripotent stem cells. a hPSCs can be aggregated at high density in scaffold-based or scaffold-free methods. b Heterogeneous cell assemblies can be constructed by merging independently differentiated

C Tissue Organization
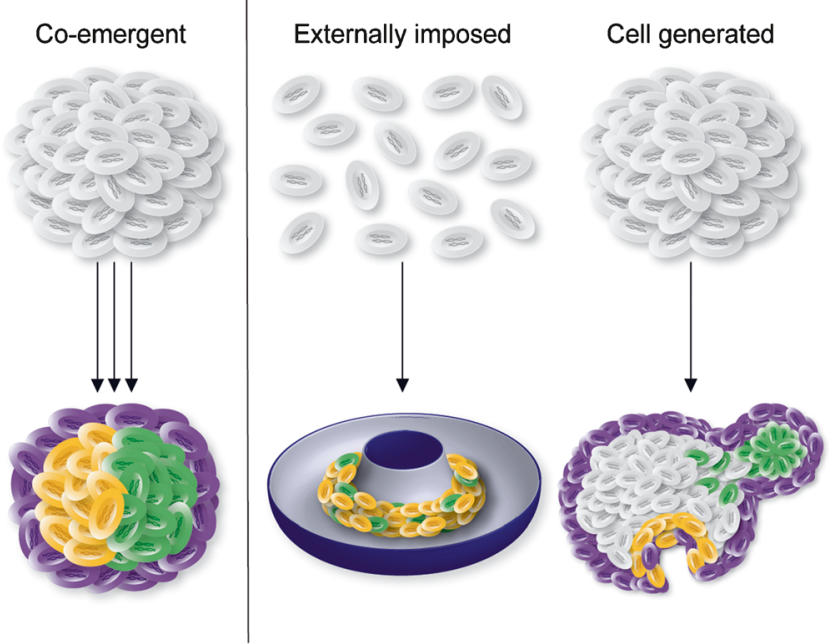

cell populations or by simultaneously co-emerging multiple differentiated phenotypes within multicellular constructs. c Higher-order tissue architecture can be achieved by externally imposed forces or by cell-generated approaches 
required to promote directed differentiation. For example, hPSCs seeded onto hydrolytically degradable $50 \%$ poly(lactic-co-glycolic acid) (PLGA) and $50 \%$ poly(L-lactic acid) (PLLA) porous scaffolds resulted in the spontaneous formation of complex multicellular structures reminiscent of primitive tissue organization. Addition of specific growth factors to the culture of hPSC-seeded scaffolds directed differentiation toward specific lineages: TGF- $\beta$-induced cartilage-like extracellular matrix (ECM) secretion, activin-A- or IGFinduced structures analogous to the developing liver, and retinoic acid (RA)-induced morphologic features of the embryonic neural tube [22]. This early study demonstrated that hPSCs can attach to synthetic scaffolds and exhibit nascent signs of differentiation in response to growth factor stimulation. However, the resulting tissue structures composed of heterogeneous cell populations highlight the need to incorporate more robust differentiation protocols with scaffold-based methods.

The use of porous scaffolds as a platform for hPSC-derived tissue formation has been studied largely in the context of chondrogenic and osteogenic differentiation [23, 24]. One explanation for this pairing is that bone and cartilage arise from the mesoderm germ layer and stiffer scaffolds have been shown to promote hPSC differentiation into the mesoderm lineage, observed through increased expression of mesoderm-specific genes, FOXF1, MEOX1, and KDR [25, 26]. Scaffolds of intermediate stiffness induce endodermal gene expression and softer scaffolds give rise to ectodermspecific gene expression [26]; therefore, modulating matrix mechanical properties can further dictate tissue form and function.

Another goal when constructing material scaffolds is to mimic aspects of the ECM specific to the model tissue. Hydrogels are often chosen for their ability to improve cell survival and enhance cell differentiation after transplantation, yet they can also induce cell-mediated remodeling in vitro. For instance, hydrogels made from pulverized and lyophilized decellularized porcine heart tissue and reconstituted with type I collagen can direct in vitro cardiac differentiation of hPSCs. Encapsulation of 3D hPSC aggregates within decellularized heart/collagen hydrogels recapitulates the collagenous nature of native heart ECM and in turn improves cardiac differentiation as seen through cell contractility, gene expression of cardiac troponin $\mathrm{T}$, and the presence of cardiac markers cardiac troponin I and connexin 43 [27]. Similarly, hESC aggregates encapsulated in dextran-based hydrogels loaded with VEGF microparticles and cell-adhesive RGD peptides enhanced vascular differentiation from hPSCs [28]. These studies demonstrate that hydrogel-based scaffolds are capable of mimicking aspects of native ECM by providing cell adhesive substrates that facilitate cell attachment, growth, and differentiation.

As described above, polymeric scaffolds function as a platform to facilitate hPSC assembly; however, the ability to instruct cell fate based on materials alone has been limited. The addition of soluble factors to the culture medium remains the primary driving force directing hPSC differentiation on the scaffolds. Moreover, the ratio of material to cells is skewed largely in favor of material rather than cells. Although in many instances polymeric scaffolds are designed to degrade and leave multicellular tissue behind, this strategy has yielded limited successes to date.

\section{Scaffold-Free hPSC Assembly}

In contrast to material-based approaches, scaffold-free assembly is dependent on the cells' collective ability to amass into a three-dimensional construct. Just as undifferentiated hPSCs grow as tightly packed colonies in monolayer culture, the epithelial phenotype of hPSCs contributes to their ability to form tightly packed three-dimensional multicellular aggregates. Endogenously driven 3D cell assembly stimulates an increase in intercellular adhesion molecules, such as cadherins and connexins [29,30]. Cell-cell contact also plays important roles in maintaining pluripotency and facilitating downstream signaling, such as the Notch pathway, that regulates stem cell maintenance and self-renewal [17].

A variety of techniques exist to initiate hPSC aggregate assembly, which differ in scale of production. The approaches range from single aggregates formed in separate vessels (individual) [31] to many aggregates in a shared volume (bulk) [32, 33]. Embryoid body culture originated with the hanging drop assembly method, which relies on gravity to force cells into close proximity within small "drops" suspended from a lid, but has been adapted in recent years to use V-bottomed or round-bottomed multiwell plates for aggregate assembly. The technical and logistic challenges associated with the manipulation of individual aggregates has often motivated the use of bulk methods for hPSC assembly since they are more convenient and inherently scalable when larger numbers of aggregates are needed or desired [34]. Different variations of microwell arrays provide robust platforms to force hPSC aggregation by centrifugation [31, 35, 36], allowing for the generation of $100 \mathrm{~s}-1000$ s of aggregates in parallel at once at high spatial density.

Scaffold-free assembly of hPSC constructs does not necessarily preclude the use of materials; however, the inclusion of materials, typically in the form of microparticles or nanoparticles, is not the primary mechanism for cell assembly. For instance, microparticles (MPs) can be included within PSC aggregates as a vehicle for molecular delivery, imaging, and external manipulations [37, 38]. Retinoic acid delivery from degradable PLGA MPs incorporated within mouse ESC aggregates induced the formation of highly organized, cystic spheroids that closely resembled mouse epiblasts [39]. Similarly, gelatin MPs embedded in mESCs and loaded with BMP4 resulted in mesoderm gene expression while noggin 
delivery induced ectoderm gene expression. Compared to soluble delivery methods, MP delivery of BMP4 reduced the amount of growth factor needed to elicit a comparable phenotypic response by an order of magnitude [40]. These examples indicate that microparticle delivery of biomolecules within scaffold-free aggregates can efficiently direct stem cell differentiation and initiate morphogenic processes. Although these proof-of-principle studies were conducted with mouse ESCs, the same methods can be directly translated to human PSC aggregates. PLGA microparticles that were embedded into human ESC aggregates promoted osteogenic differentiation when loaded with BMP2 or endothelial differentiation when loaded with VEGF [41]. Therefore, using polymer microparticles and nanoparticles within scaffold-free constructs provides a novel means to control the spatiotemporal effects of morphogenic factors on cell specification and tissue formation.

\section{Inducing Phenotypic Heterogeneity Within Multicellular Constructs}

While multicellularity provides the starting basis for tissue formation, the cooperative interactions of organized groups of cells contribute to complex tissue function(s). The presence of two or more cell types within tissues largely arises from a need for mutually supportive co-dependence. For example, cardiac fibroblasts support the function of cardiomyocytes by synthesizing the majority of the extracellular matrix proteins in the heart, secreting mitogens and cytokines that promote cardiomyocyte growth and survival, and electrically coupling with cardiomyocytes [42]. Similarly, oligodendrocytes in vivo act as a support system for neurons by myelinating axons [43], while electrically active neurons regulate oligodendrocyte development by controlling oligodendrocyte precursor cell proliferation, differentiation, and myelin biosynthesis [44].

A defined, yet heterogeneous, tissue construct of hPSCderived cells can be attained using two fundamentally different strategies, denoted here as (1) merging of independently differentiated cell populations or (2) co-emergence of differentiated phenotypes. Pluripotent cells can be independently differentiated from a single source of hPSCs into specific cell types in parallel and combined after differentiation (i.e., merged populations). Alternatively, multiple cell types can be differentiated simultaneously in the same culture to form a heterotypic, multicellular aggregate from a single starting population of hPSCs (i.e., co-emergence; Fig. 1b).

\section{Merging of Independently Differentiated Cell Populations}

The incorporation of multiple cell types in engineered tissues has been motivated primarily by the need for vascularization of dense multicellular constructs. Inclusion of endothelial cells enables the formation of vascular networks to assist with nutrient diffusion throughout engineered tissues. Additionally, stromal cell populations support vascular networks, provide paracrine signals to surrounding cells, and secrete extracellular matrix that supports cell functions and tissue structure, making stromal cells a very desirable population to include in engineered tissues. The mixing of multiple cell phenotypes is highlighted in the generation of human cardiac tissue patches. Engineered patches comprised of hESC-derived cardiomyocytes (hESC-CMs), human umbilical vein endothelial cells (HUVECs), and human dermal fibroblasts (HDFs) contained more vessel structures and formed significantly larger grafts in vivo compared to patches comprised solely of hESC-CMs, which survived poorly upon transplantation [45]. Similarly, iPSC-derived hepatic endoderm cells (iPSCHEs) cultured with HUVECs and human mesenchymal stem cells (hMSCs) condensed into three-dimensional liver buds (iPSC-LBs) that not only connected with host vasculature promptly following transplantation but also established an instructive vascular niche, allowing for liver organogenesis and regeneration $[46 \bullet \bullet, 47]$. These examples demonstrate that hPSC-derived tissue function can be enhanced with heterotypic constructs that contain vascular and stromal cell populations.

Methods to merge independently differentiated cell types include both extrinsically driven scaffold-based platforms [48-50, 51••] and scaffold-free approaches [46••, 47, 52]. Most studies to date have relied on primary isolated cells mixed with a single defined hPSC-derived cell type. However, with improved differentiation protocols for an increasing number of cell types, it is now feasible to obtain multiple distinct cell types from the same starting pluripotent population. A recent example of this principle is described in the generation of cardiac cell sheets. Human iPSCs were differentiated into cardiomyocytes (CMs), endothelial cells (ECs), and mural cells (MCs). The differentiated cell populations were mixed and plated on poly(N-isopropylacrylamide) (PIPAAm) polymer surfaces, where they assembled robust beating cell sheets that were composed entirely of hPSCderived cells $[51 \bullet \bullet]$.

One advantage of merging distinct populations is that the starting stoichiometries of the independently differentiated populations can be precisely controlled at the outset. Altering the ratios of different initial cell populations is the most direct means of varying the proportions of cells within the resulting tissues. However, an important consideration is the relative proliferative capacity of each cell type within the system. Combining cell types with drastically different proliferation rates could lead to outcomes where the ratios of cell types at the input of the experiment differ dramatically from the ratio of cells in the final functional tissue. In addition to optimizing cell ratios, identifying culture media and other 
components that mutually support each individual cell population is also critical to the formation of functional tissue constructs.

\section{Co-emergence of Specified Cell Populations}

In contrast to merging distinct cell populations, aggregates of pluripotent stem cells can support co-emergence of multiple cell types given the proper instructive cues. The recent series of organoid formation studies demonstrates that temporal regulation of biochemical or environmental cues in an hPSC starting population can produce in vitro models of human organ development, including defined regions of cortical tissue, intestine, retina, and kidney [14-16, 53].

While cellular remodeling and organization during organoid formation is largely autonomously mediated, developing effective organoid formation strategies remains an iterative process. Intercellular crosstalk and feedback mechanisms during organoid formation are relatively unknown; therefore, protocol evolution involves a systematic trial-and-error approach. For example, during optic cup development, the Wnt agonist CHIR99021 induces retinal pigment epithelium (RPE) formation only when added after cellular commitment to the retinal fate. However, since neural retina (NR) tissue in addition to RPE tissue is important for optic cup organization, the temporal effects of adding CHIR99021 on tissue differentiation was examined so as to induce simultaneous differentiation of RPE and NR tissues $[15 \cdot \bullet]$. Similarly, serial addition of defined growth factors to hPSCs resulted in a step-wise induction of phenotypes that mimicked embryonic intestinal development. ActivinA treatment directed hPSC differentiation to definitive endoderm, while subsequent treatment with WNT3A and FGF4 promoted hindgut and intestinal specification, and further culture in pro-intestinal culture conditions initiated the development of hindgut spheroids into intestinal organoids in a manner that mimicked fetal gut development [53].

Therefore, it is evident that the co-emergence approach is very much an outcome-driven process. Culture conditions for pluripotent aggregates are manipulated until the desired outcome (or a progenitor of the desired outcome) is achieved. Comparison to the strategy of merging independently differentiated populations reveals a level of control that is lacking in the initial stages of organoid culture in that specific cell ratios and cell types are not specifically pre-determined. Despite being perhaps less well-defined at the outset, co-emergent protocols reproducibly result in functional tissue constructs where all cell types come from the same starting cell population, thereby more accurately mimicking in vivo embryonic developmental processes.

\section{Directing Higher-Order Organization and Tissue Architecture}

An additional level of organization within engineered tissues involves structurally assembling cells into specific multicellular arrangements that are analogous to the architecture observed in the native tissues that they are intended to model. While higher-order assembly can be achieved in many ways, most approaches can be categorized as either exogenously imposed technologies which rely on molds or scaffolds to dictate tissue shape or endogenously driven morphogenesis in which self-organization mechanisms yield patterning without external physical guidance or instruction (Fig. 1c).

\section{Externally Imposed Tissue Architecture}

The most common approach for generating higher-order tissue architecture has been to use engineered molds or scaffolds to dictate the shape of the tissue. One advantage of such techniques is the ability to control and define the ultimate meso/ macroscopic size and shape of the tissues. Scaffolds can be used as a means to not only assemble cells together into multicellular constructs but also provide dimensionality specific to the tissue being modeled. Decellularized tissues have been examined as scaffolds given that they already possess the native shape and geometry of original tissues of interest. Repopulation of decellularized tissues has been examined by the perfusion of human pluripotent derived cell populations through decellularized lung [54, 55], heart [56, 57], and kidney [58]. However, retaining bioactive extracellular matrix components throughout the decellularization process and repopulating acellular scaffolds with sufficient cell densities and cell types to achieve functional tissues remain challenges in this field.

As an alternative to pre-determined scaffold shape to control tissue architecture, externally directed tissue geometries can be induced by seeding cells into physical molds that impose mechanical constraints to force assembly in particular configurations. This strategy is based on collective cell remodeling to the lowest energy state permissible given the specific boundary conditions defined by a particular mold geometry [59]. Molds can be generated from a variety of materials including agarose, polydimethylsiloxane, or polyacrylamide that have minimal adhesive interactions with the cells and serve mainly as a means of aggregating cells and forcing tissue shape $[35,59,60]$. Spheroidal shapes are the lowest energy state achieved by multicellular constructs and therefore are the easiest and most common shape to generate. Scientists have frequently exploited this concept by producing basic molds that force cells to aggregate into periodically spaced wells, where cell-cell adhesions drive self-assembly and condensation into spheroids [30]. The resulting cell aggregates retain three-dimensional architecture and have been generated from hPSCs or hPSC-derived cells 
to model embryonic development of specific tissues. Additionally, small aggregates can be used as building blocks to generate larger, more complex tissues through manual manipulation or bioprinting techniques [61].

Generation of more complex shapes can also be achieved by producing more complex mold geometries. However, the final shape of the tissue is not dictated by physical mold boundaries but is mediated by cell-induced tensile forces. Therefore, molds often include elements such as posts, which physically prevent the tissue from remodeling into spheroids and maintain tissues under tension $[62,63]$. This approach may be limited, however, to tissues where cellular tension is inherently found to promote tissue function (i.e., myocardium, ligament, and tendon) and not applicable to tissues that require formation of alternative structures like epithelial sheets.

Directing tissue architecture using molding and shaping technologies enables researchers to build three-dimensional constructs of physiologically relevant size and shape. This "top-down" approach allows the user to customize input system parameters to various design specifications. In contrast, a "bottom-up" approach that exploits cell-driven mechanisms for tissue formation provides an alternative method for achieving higher-order structure and organization within tissues.

\section{Cell-Generated Tissue Organization}

In recent years, numerous reports of hPSC-derived organoids have highlighted the ability to exploit endogenous cell mechanisms to achieve well-defined multicellular architecture within tissue constructs [14-16]. This cell-programmed approach relies upon intrinsic pathways to direct a series of cellular responses such as proliferation, collective migration, and extracellular matrix assembly and remodeling to achieve tissue-specific geometries. Examples of this can be seen in the budding of organoids, lumen formation, branching of vessels, and folding of tissue sheets [15••, 64].

Although relying upon cell-driven approaches to shape tissue structure may seem less predictable and controlled, identification of proper input cues to the system can enable hPSC morphogenesis to be highly reproducible in terms of spatial and temporal organization. This suggests that mechanisms which drive morphogenic changes act in concert with one another and are able to not only co-regulate the emergence of multiple cell populations in parallel but also balance the appropriate interactions to coordinately develop architecture in a robust manner. The consequences of multicellular interactions are readily apparent during in vitro optic cup development. A bistable environment that is permissive to growth, maturation, and survival of both the retinal pigment epithelial tissues and the neural retina is required for these two critical cell types to work together [15••]. Each cell type can activate necessary paracrine signaling pathways to maintain their phenotype and function while simultaneously inhibiting opposing signaling pathways and providing negative feedback to the overall system. Within the context of optic cup formation, Wnt signaling drives the formation of retinal pigment epithelium, whereas Wnt inhibitors secreted by neural retina attenuate Wnt activity within the neural retina, allowing both cell types to develop and function simultaneously [65••]. Higherorder structure that is achieved by independent cell types can be observed in several other organoid examples, including cortical tissues and pituitary tissues $[14,15 \bullet \cdot]$.

Cell autonomous driven methods to define tissue architecture afford the opportunity to gain insight into naturally arising biophysical cues that are associated with developmental morphogenesis. Information about cellular processes can be integrated into computational models to help understand the specific forces that guide self-directed morphogenesis. For example, developing epithelial sheets maintain smooth curvature on both the apical and basal surfaces despite cellular changes, such as proliferation, that could cause a disruption in force balance. To compensate for these local force changes in cell sheets, one model predicts that a tangential contractile force is maintained along the apical surface that reduces the impact of any irregular local force changes [66-68]. Computational predictions of cell behaviors during tissue organization not only provide insight into developmental morphogenesis but also describe tissue-level changes that occur during disease. For example, intestinal crypt fission is based on a budding process that originates from an intestinal organoid. One proposed mechanism for this morphogenesis is that deformations in the tissue initiate from heterogeneous viscoelastic properties of cells within the crypt that initiate buckling and lead to the budding process [69]. With careful analysis of the dynamics with which budding takes place and the sequential nature of continued budding from a single intestinal organoid, it is possible to predict the growth rate of intestinal epithelium and correlate it to growth of intestinal polyps [70].

\section{Conclusions}

Opportunities remain to improve upon the current approaches for constructing in vitro human tissue models. To induce tissue formation, three levels of tissue organization need to be addressed - multicellular cell assembly, heterogeneity of appropriate cell types, and higher-order tissue architecture. While this review focuses on different approaches to achieve these levels of organization, most current approaches cannot be classified into one category. The strategies discussed throughout this review define the extremes of the spectrum, whereas many current methods for hPSC tissue generation encompass aspects of each approach.

Many methods have been explored as a means to engineer human tissue constructs from pluripotent stem cells; however, fewer approaches exist for fully characterizing tissue structure 
and function. Visualization of larger engineered tissues has been limited by traditional microscopy approaches that work well for two-dimensional phenotypic and morphogenic analyses. However, recent advancements with clearing techniques [71] and lightsheet microscopy [72] allow visualization at much greater tissue depths (millimeter scale), enabling studies of spatio-temporal changes during morphogenic processes to be observed in dense three-dimensional tissues.

In addition to visualizing physical structure, mechanical characterization of engineered hPSC tissues warrants further investigation. Methods exist to examine mechanical forces at the individual cell or sub-cellular level (atomic force microscopy) or at the larger, meso-scale tissue level (tensile testing); however, measuring mechanical properties of micron-scale engineered tissue constructs that lie in between these techniques remains a challenge. To address this gap, the creation of novel systems is needed that enable measurements of bulk physical properties of multicellular aggregates [73], or contraction of small-scale tissue constructs [74]. Such methods provide direct force measurements based on beam or postdeflection because tissues at this size scale $(\mu \mathrm{m})$ makes them difficult to manually manipulate and mount on conventional force transducers.

Electrophysiology is largely used to characterize function of electrically excitable cell types, such as cardiac myocytes and neurons. However, while electrophysiology techniques exist for characterization of single cells (patch clamp) and monolayers (microelectrode arrays), there is a lack of methods to acquire similar physiological measurements of 3D meso-scale tissue constructs. As more robust methods for small-scale tissue characterization are developed, scientists in the field can work toward adopting better ex vivo standards for assessing the functional properties of 3D human tissue constructs.

Robust development of hPSC-derived tissue constructs will have tremendous impacts in the bioengineering and medical fields. Functional human tissue models ex vivo will provide novel platforms for drug screening and disease modeling that could be adapted for personalized and precision medical therapies in the future.

Acknowledgments This work was supported by funding from the California Institute for Regenerative Medicine (LA1-08015) and the National Science Foundation (NSF) (CBET 0939511). T.A.H. is currently supported by an American Heart Association (AHA) Postdoctoral Fellowship. The authors would like to thank Amy Foley-De Silva for figure illustrations.

\section{Compliance with Ethical Standards}

Conflict of Interest Oriane B. Matthys, Tracy A. Hookway, and Todd C. McDevitt declare that they have no conflict of interest.

Human and Animal Rights and Informed Consent This article does not contain any studies with human or animal subjects performed by any of the authors.

\section{References}

Papers of particular interest, published recently, have been highlighted as:

•• Of major importance

1. Thomson JA et al. Embryonic stem cell lines derived from human blastocysts. Science. 1998;282(5391):1145-7.

2. Yu J et al. Induced pluripotent stem cell lines derived from human somatic cells. Science. 2007;318(5858):1917-20.

3. Takahashi $\mathrm{K}$ et al. Induction of pluripotent stem cells from adult human fibroblasts by defined factors. Cell. 2007;131(5):861-72.

4. Lian X et al. Robust cardiomyocyte differentiation from human pluripotent stem cells via temporal modulation of canonical Wnt signaling. Proc Natl Acad Sci U S A. 2012;109(27):E1848-57.

5. Lian X et al. Directed cardiomyocyte differentiation from human pluripotent stem cells by modulating Wnt/beta-catenin signaling under fully defined conditions. Nat Protoc. 2013;8(1):162-75.

6. Laflamme MA et al. Cardiomyocytes derived from human embryonic stem cells in pro-survival factors enhance function of infarcted rat hearts. Nat Biotechnol. 2007;25(9):1015-24.

7. Wichterle $\mathrm{H}$ et al. Directed differentiation of embryonic stem cells into motor neurons. Cell. 2002;110(3):385-97.

8. Yan Y et al. Directed differentiation of dopaminergic neuronal subtypes from human embryonic stem cells. Stem Cells. 2005;23(6): 781-90.

9. Idelson $\mathrm{M}$ et al. Directed differentiation of human embryonic stem cells into functional retinal pigment epithelium cells. Cell Stem Cell. 2009;5(4):396-408.

10. Song $\mathrm{Z}$ et al. Efficient generation of hepatocyte-like cells from human induced pluripotent stem cells. Cell Res. 2009;19(11): 1233-42.

11. Cai J et al. Directed differentiation of human embryonic stem cells into functional hepatic cells. Hepatology. 2007;45(5):1229-39.

12. Zhang D et al. Highly efficient differentiation of human ES cells and iPS cells into mature pancreatic insulin-producing cells. Cell Res. 2009;19(4):429-38.

13. D'Amour KA et al. Production of pancreatic hormone-expressing endocrine cells from human embryonic stem cells. Nat Biotechnol. 2006;24(11):1392-401.

14. Lancaster MA et al. Cerebral organoids model human brain development and microcephaly. Nature. 2013;501(7467):373-9.

15.• Nakano T et al. Self-formation of optic cups and storable stratified neural retina from human ESCs. Cell Stem Cell. 2012;10(6):77185. This study demonstrates that human ESCs can self-organize in vitro to generate an optic cup structure that contains rods, cones, and photoreceptors in the multilayered human tissue.

16. Xia Y et al. The generation of kidney organoids by differentiation of human pluripotent cells to ureteric bud progenitor-like cells. Nat Protoc. 2014;9(11):2693-704.

17. Teo A, Lim M, Weihs D. Embryonic stem cells growing in 3dimensions shift from reliance on the substrate to each other for mechanical support. J Biomech. 2015;48(10):1777-81.

18. Schaller MD. Cellular functions of FAK kinases: insight into molecular mechanisms and novel functions. J Cell Sci. 2010;123(Pt 7): 1007-13.

19. Shao Y, Sang J, Fu J. On human pluripotent stem cell control: the rise of $3 \mathrm{D}$ bioengineering and mechanobiology. Biomaterials. 2015;52:26-43.

20. Lu HF et al. A 3D microfibrous scaffold for long-term human pluripotent stem cell self-renewal under chemically defined conditions. Biomaterials. 2012;33(8):2419-30.

21. Carlson AL et al. Microfibrous substrate geometry as a critical trigger for organization, self-renewal, and differentiation of human 
embryonic stem cells within synthetic 3-dimensional microenvironments. FASEB J. 2012;26(8):3240-51.

22. Levenberg $\mathrm{S}$ et al. Differentiation of human embryonic stem cells on three-dimensional polymer scaffolds. Proc Natl Acad Sci U S A. 2003;100(22):12741-6.

23. Jin GZ et al. Bone tissue engineering of induced pluripotent stem cells cultured with macrochanneled polymer scaffold. J Biomed Mater Res A. 2013;101(5):1283-91.

24. Wang $\mathrm{P}$ et al. Bone tissue engineering via human induced pluripotent, umbilical cord and bone marrow mesenchymal stem cells in rat cranium. Acta Biomater. 2015;18:236-48.

25. Evans ND et al. Substrate stiffness affects early differentiation events in embryonic stem cells. Eur Cell Mater. 2009;18:1-13. discussion 13-4.

26. Zoldan $\mathrm{J}$ et al. The influence of scaffold elasticity on germ layer specification of human embryonic stem cells. Biomaterials. 2011;32(36):9612-21.

27. Duan $Y$ et al. Hybrid gel composed of native heart matrix and collagen induces cardiac differentiation of human embryonic stem cells without supplemental growth factors. J Cardiovasc Transl Res. 2011;4(5):605-15.

28. Ferreira LS et al. Bioactive hydrogel scaffolds for controllable vascular differentiation of human embryonic stem cells. Biomaterials. 2007;28(17):2706-17.

29. Chen $\mathrm{T}$ et al. E-cadherin-mediated cell-cell contact is critical for induced pluripotent stem cell generation. Stem Cells. 2010;28(8): 1315-25.

30. Bao B et al. Connexon-mediated cell adhesion drives microtissue self-assembly. FASEB J. 2011;25(1):255-64.

31. Ungrin MD et al. Reproducible, ultra high-throughput formation of multicellular organization from single cell suspension-derived human embryonic stem cell aggregates. PLoS One. 2008;3(2):e1565.

32. Abbasalizadeh $\mathrm{S}$ et al. Bioprocess development for mass production of size-controlled human pluripotent stem cell aggregates in stirred suspension bioreactor. Tissue Eng Part C Methods. 2012;18(11): 831-51.

33. Otsuji TG et al. A 3D sphere culture system containing functional polymers for large-scale human pluripotent stem cell production. Stem Cell Rep. 2014;2(5):734-45.

34. Kurosawa $\mathrm{H}$. Methods for inducing embryoid body formation: in vitro differentiation system of embryonic stem cells. J Biosci Bioeng. 2007;103(5):389-98.

35. Bauwens CL, Ungrin MD. Scalable cardiac differentiation of human pluripotent stem cells as microwell-generated, size controlled three-dimensional aggregates. Methods Mol Biol. 2014;1181:1525.

36. Pettinato $\mathrm{G}$ et al. ROCK inhibitor is not required for embryoid body formation from singularized human embryonic stem cells. PLoS One. 2014;9(11):e100742.

37. Bratt-Leal AM et al. Incorporation of biomaterials in multicellular aggregates modulates pluripotent stem cell differentiation. Biomaterials. 2011;32(1):48-56.

38. Bratt-Leal AM et al. Magnetic manipulation and spatial patterning of multi-cellular stem cell aggregates. Integr Biol (Camb). 2011;3(12):1224-32.

39. Carpenedo RL et al. Homogeneous and organized differentiation within embryoid bodies induced by microsphere-mediated delivery of small molecules. Biomaterials. 2009;30(13):2507-15.

40. Bratt-Leal AM et al. A microparticle approach to morphogen delivery within pluripotent stem cell aggregates. Biomaterials. 2013;34(30):7227-35.

41. Qutachi O, Shakesheff KM, Buttery LD. Delivery of definable number of drug or growth factor loaded poly(DL-lactic acid-coglycolic acid) microparticles within human embryonic stem cell derived aggregates. J Control Release. 2013;168(1):18-27.
42. Ottaviano FG, Yee KO. Communication signals between cardiac fibroblasts and cardiac myocytes. J Cardiovasc Pharmacol. 2011;57(5):513-21.

43. Simons M, Trajkovic K. Neuron-glia communication in the control of oligodendrocyte function and myelin biogenesis. J Cell Sci. 2006;119(Pt 21):4381-9.

44. Gibson EM et al. Neuronal activity promotes oligodendrogenesis and adaptive myelination in the mammalian brain. Science. 2014;344(6183):1252304.

45. Stevens KR et al. Physiological function and transplantation of scaffold-free and vascularized human cardiac muscle tissue. Proc Natl Acad Sci U S A. 2009;106(39):16568-73.

46.• Takebe $\mathrm{T}$ et al. Vascularized and functional human liver from an iPSC-derived organ bud transplant. Nature. 2013;499(7459):481-4. This article is one of the first reports demonstrating the generation of a functional human organ from iPSCs by the transplantation of liver buds created in vitro.

47. Takebe $\mathrm{T}$ et al. Vascularized and complex organ buds from diverse tissues via mesenchymal cell-driven condensation. Cell Stem Cell. 2015;16(5):556-65.

48. Levenberg $\mathrm{S}$ et al. Engineering vascularized skeletal muscle tissue. Nat Biotechnol. 2005;23(7):879-84.

49. Lesman A et al. Transplantation of a tissue-engineered human vascularized cardiac muscle. Tissue Eng Part A. 2010;16(1):11525 .

50. Takebe T et al. Generation of functional human vascular network Transplant Proc. 2012;44(4):1130-3.

51.• Masumoto H et al. Human iPS cell-engineered cardiac tissue sheets with cardiomyocytes and vascular cells for cardiac regeneration. Sci Rep. 2014;4:6716. This report simultaneously differentiates cardiomyocytes and vascular cells from human iPSCs to generate beating cardiac tissue sheets that rescue cardiac function in infarcted rat hearts.

52. Matsumoto $\mathrm{K}$ et al. Liver organogenesis promoted by endothelial cells prior to vascular function. Science. 2001;294(5542):559-63.

53. Spence JR et al. Directed differentiation of human pluripotent stem cells into intestinal tissue in vitro. Nature. 2011;470(7332):105-9.

54. Gilpin SE et al. Enhanced lung epithelial specification of human induced pluripotent stem cells on decellularized lung matrix. Ann Thorac Surg. 2014;98(5):1721-9. discussion 1729.

55. Ghaedi $\mathrm{M}$ et al. Human iPS cell-derived alveolar epithelium repopulates lung extracellular matrix. J Clin Invest. 2013;123(11): 4950-62.

56. Lu TY et al. Repopulation of decellularized mouse heart with human induced pluripotent stem cell-derived cardiovascular progenitor cells. Nat Commun. 2013;4:2307.

57. Carvalho, J.L., et al., Characterization of Decellularized Heart Matrices as Biomaterials for Regular and Whole Organ Tissue Engineering and Initial Recellularization with Ips Cells. J Tissue Sci Eng, 2012. Suppl 11: p. 002.

58. Caralt $\mathrm{M}$ et al. Optimization and critical evaluation of decellularization strategies to develop renal extracellular matrix scaffolds as biological templates for organ engineering and transplantation. Am J Transplant. 2015;15(1):64-75.

59. Youssef J et al. Quantification of the forces driving self-assembly of three-dimensional microtissues. Proc Natl Acad Sci U S A. 2011;108(17):6993-8.

60. Dahlmann J et al. The use of agarose microwells for scalable embryoid body formation and cardiac differentiation of human and murine pluripotent stem cells. Biomaterials. 2013;34(10):2463-71.

61. Faulkner-Jones A et al. Bioprinting of human pluripotent stem cells and their directed differentiation into hepatocyte-like cells for the generation of mini-livers in 3D. Biofabrication. 2015;7(4):044102.

62. Tejavibulya $\mathrm{N}$ et al. Directed self-assembly of large scaffold-free multi-cellular honeycomb structures. Biofabrication. 2011;3(3): 034110 . 
63. Bian $\mathrm{W}$ et al. Robust T-tubulation and maturation of cardiomyocytes using tissue-engineered epicardial mimetics. Biomaterials. 2014;35(12):3819-28.

64. Kusuma $\mathrm{S}$ et al. Self-organized vascular networks from human pluripotent stem cells in a synthetic matrix. Proc Natl Acad Sci U S A. 2013;110(31):12601-6.

65.• Sasai Y. Cytosystems dynamics in self-organization of tissue architecture. Nature. 2013;493(7432):318-26. This review highlights the self-assembly, self-patterning, and self morphogenesis processes of tissue self-organization and describes the mechanisms involved in each mode of tissue self-organization.

66. Okuda $\mathrm{S}$, et al. Modeling cell apoptosis for simulating threedimensional multicellular morphogenesis based on a reversible network reconnection framework. Biomech Model Mechanobiol. 2015.

67. Okuda $\mathrm{S}$ et al. Apical contractility in growing epithelium supports robust maintenance of smooth curvatures against cell-divisioninduced mechanical disturbance. J Biomech. 2013;46(10):170513.
68. Okuda $\mathrm{S}$ et al. Reversible network reconnection model for simulating large deformation in dynamic tissue morphogenesis. Biomech Model Mechanobiol. 2013;12(4):627-44.

69. Pin C, Watson AJ, Carding SR. Modelling the spatio-temporal cell dynamics reveals novel insights on cell differentiation and proliferation in the small intestinal crypt. PLoS One. 2012;7(5):e37115.

70. Pin $\mathrm{C}$ et al. An individual based computational model of intestinal crypt fission and its application to predicting unrestrictive growth of the intestinal epithelium. Integr Biol (Camb). 2015;7(2):213-28.

71. Zhu D et al. Recent progress in tissue optical clearing. Laser Photonics Rev. 2013;7(5):732-57.

72. Wolenski JS, Julich D. Fluorescence microscopy gets faster and clearer: roles of photochemistry and selective illumination. Yale J Biol Med. 2014;87(1):21-32.

73. Kinney MA, Saeed R, McDevitt TC. Mesenchymal morphogenesis of embryonic stem cells dynamically modulates the biophysical microtissue niche. Sci Rep. 2014;4:4290.

74. Boudou $\mathrm{T}$ et al. A microfabricated platform to measure and manipulate the mechanics of engineered cardiac microtissues. Tissue Eng Part A. 2012;18(9-10):910-9. 